\title{
Antitubercular and Antimicrobial Activity of Some Oxoquinoline Derivatives
}

\author{
S. P. SHRIVASTAVA, RACHANA RAI* and KEERTHI KUMAR KODARI \\ Department of Chemistry, Heterocyclic Research Laboratory, \\ Dr. Hari Singh Gour University, Sagar (M.P)-470003, India \\ rachanaraiphd6@gmail.com
}

Received 4 July 2012 / Accepted 28 July 2012

\begin{abstract}
Different derivatives of (Z)-1-(4'-(benzylidineamino)-[1,1'-biphenyl]-4-yl)-5,7-dihydroxy4-methyl-1,8a-dihydroquinolin-2(3H)-one have been prepared and all the compounds have been characterized by IR, ${ }^{1} \mathrm{H}$ NMR, Mass and elemental analysis. In vitro-anti-tubercular activity was carried out against mycobacterium tuberculosis $\mathrm{H}_{37} \mathrm{RV}$ strain and antimicrobial activity against various bacteria and fungi.
\end{abstract}

Keywords: Phloroglucinal, Ethylacetoacetate, Benzidine, Anti-tuberculosis

\section{Introduction}

Now a day's TB is a major global health problem and is the second leading cause of death from an infectious disease worldwide, by the WHO report there were an estimated 8.5-9.2 million cases and 1.2-1.5 million deaths, by this figure we understand the seriousness of TB and it is a contagious disease for both animals and humans, caused by bacilli belonging the mycobacterium tuberculosis complex, in the majority of the cases TB is due to mycobacterium tuberculosis (Koch's bacillus). M.tuberculosis strains showing good resistance against isoniazid ${ }^{1-2}$. Oxoquinooline moiety is an important scaffold known to be reported with several biological aspects antiseptic, analgesic, trypanocidal, germicidal, antitubercular $^{3}$ and antitumor activity ${ }^{4-9}$, anti malarial agents ${ }^{10-13}$ amoebiudal, antihypertensive, anti cancer, analgesic and curareform activities ${ }^{14-17}$, antitumor activities ${ }^{18}$ oxoquinoline nucleus posses a variety of biological activity ${ }^{19-20}$.

\section{Experimental}

A mixture of phloroglucinal ( 0.1 mole) and ethylacetoacetate $(0.1$ mole) with $70 \%$ sulfuric acid was refluxed up to $30 \mathrm{~min}$. The resulting dark green solution was cooled and poured into crushed ice, the crude product was filtered off and washed repeatedly with water, the resulting product was dried and crystallized with methanol. The reaction progress was monitored by TLC using ethylacetate: hexane (1:1) as a mobile phase. 
Synthesis of 1-(4'-amino-[1,1'-biphenyl]-4-yl)-5,7-dihyydroxy-4-methyl-1,8a-dihydroquinolin-2(3H)one

Mixture of 5,7-dihydroxy-4-methyl-3,8a-dihydro- $2 \mathrm{H}$-chromen-2-one ( $0.1 \mathrm{~mole})$ and benzidine $(0.1$ mole $)$ in anhydrous pyridine was refluxed for $4 \mathrm{~h}$ under anhydrous conditions. Subsequently the resultant reaction mixture was acidified with dil. $\mathrm{HCl}$ and poured into ice cold water. A solid separated out was filtered off successively with water and purified by crystallization from aqueous methanol.

Synthesis of (Z)-1-(4'-(benzylidineamino)-[1,1'-biphenyl]-4-yl)-5,7-dihydroxy-4methyl-1,8a-dihydroquinolin-2(3H)-one

A mixture of 1-(4'-amino-[1,1'-biphenyl]-4-yl)-5,7-dihyydroxy-4-methyl-1,8a-dihydroquinolin$2(3 \mathrm{H})$ one $(0.1 \mathrm{~mole})$ and 0.1 mole of aryl aldehyde in absolute ethanol $(30 \mathrm{~mL})$ in presence of glacial acetic acid $(1 \mathrm{~mL})$ was refluxed for 8-10 h. Excess of solvent was removed under reduced pressure. The solid obtained was washed with cold water, several times and crystallized from methanol (Scheme 1).

\section{Results and Discussion}

Final compounds were characterized by IR, NMR and elemental analysis. The MIC of synthesized compound was carried out by broth micro dilution method as described by $\operatorname{rattan}^{21}$. All compounds were tested for antibacterial activity and that found good to moderate activity. The synthesized oxoquinoline derivatives III(c), III(d) and III(h) are highly active against selected bacteria. Compounds III(a), III((b) III(e), III(g) and III(j) are moderately active and compounds III(f), III(i), III(k) and III(l) showed weakly active on selected bacteria. Compounds III(h), III(J) and III(i), showed high antifungal activity against selected fungus. Compounds III(a), III(b) and III(k) showed good to moderate activity and other compounds showed normal activity. It was also observed that the promising antimicrobials have proved to be better anti-tubercular activity. Specially compounds III $(\mathrm{h})>\operatorname{III}(\mathrm{g})>\operatorname{III}(\mathrm{a})>\operatorname{III}(\mathrm{e})>\operatorname{III}(\mathrm{C})$, due to their better activity against $\mathrm{H}_{37} \mathrm{RV}$ strain are the best choice for the preparation of new derivative in order to improve anti-tubercular activity in future. Elemental analysis of synthesized compounds has been shown in Table 1.<smiles>Oc1cc(O)cc(O)c1</smiles>

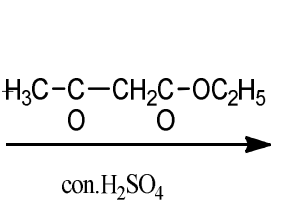$$
\mathrm{CO}_{2} \mathrm{~S}_{4}
$$<smiles>CCn1c(=O)cc(C)c2c(O)cc(O)cc21</smiles><smiles>[R]c1ccccc1/C=N\c1ccc(-c2ccc(N=O)cc2)cc1</smiles>

(I)

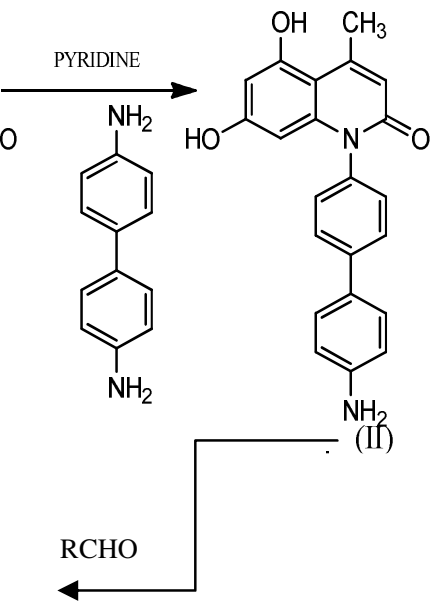<smiles>C=C1C=C(C)c2c(O)cc(O)cc2O1</smiles>

Scheme 1 


\section{IR spectral data of the compounds}

IR ( $v_{\max }$ in $\mathrm{cm}^{-1)}$ III(a): 3281.02 (O-H Str), $3039.91 \mathrm{Ar}$ (C-H str.), 2953.12 Ali (C-H str.), 1602.9 (C=O str.), 1247.99 (C=N str.), 825.56 (C-N str.), 1080.17 (C-O-C str.), 1454.38(C$\mathrm{H}$ bending in plane), 693.4 (C-H bending out of plane).

IIII: 3340.82 (O-H str), 3070.78 Aro (C-H str), 2902.96 Ali (C-H str.), 1662.69 $(\mathrm{C}=$ Ostr. $), 1139.75(\mathrm{C}=\mathrm{N}), 1288.49(\mathrm{C}-\mathrm{N}), 761.91(\mathrm{Aro}-\mathrm{Cl}), 1394.58(\mathrm{C}-\mathrm{H}$ bending in plane $)$, 698.25(C-H bending out of plane).

III(e) : $3267.52(\mathrm{O}-\mathrm{H}), 3143$ Aro $(\mathrm{C}-\mathrm{H}), 2860.53$ Ali $(\mathrm{C}-\mathrm{H}), 1155.4(\mathrm{C}=\mathrm{N}), 1348.29(\mathrm{C}-\mathrm{N})$, $1681.98(\mathrm{C}=\mathrm{O}), 1560.46(\mathrm{C}-\mathrm{O}), 1398.44$ (C-H bending in plane), 830.74 (C-H bending out of plane).

III (g) : 3211.59 (O-H), 3076.56 Aro (C-H), 1558.54 (C-O), 2964.69 Ali (C-H). 1193.98 $(\mathrm{C}=\mathrm{N}), 1361.79(\mathrm{C}-\mathrm{N}) 1683.91(\mathrm{C}=\mathrm{O}), 1126.47$ (C-O-C), 1398.44(C-H bending in plane), 821.7(C-H bending out of plane).

III(h) : 3296.46(O-H Str), 3032.2, Aro(C-H), 2922.25, Ali (C-H), $1638.91(\mathrm{C}=\mathrm{O})$, $1286.56(\mathrm{C}=\mathrm{N}), 1350.22(\mathrm{C}-\mathrm{N}), 1558.54(\mathrm{C}-\mathrm{O}), 1489.1(\mathrm{C}-\mathrm{H}$ bending in plane $), 567.09(\mathrm{C}-\mathrm{H}$ bending out of plane)

\section{${ }^{1} \mathrm{H} N M R$ spectral data}

$\left(\mathrm{CDCl}_{3}\right.$ in ppm) III(a) : 6.625(Sym. Multi, $\mathrm{OCH}_{3}$ substituted benzene ring 4H), 6.002(Sym. Multi, 2 sub benzene rings, $8 \mathrm{H}$ ), 6.428(unsym multi, hydroxy subs ring oxquinoline), 2.441 $\left(\mathrm{S}, 3 \mathrm{H}, \mathrm{CH}_{3}\right) 9.251(\mathrm{~S}, 2 \mathrm{H}, 2 \mathrm{OH})$.

III(g): 6.772(unsym multiplet, (3H, OH methoxy sub benzene ring) 9.013(S, 3H, 3-OH), $6,425(3 \mathrm{H}$, unsym. Multiplet), hydroxy sub oxoquinoline ring), 3.448 ( $\mathrm{S}, 3 \mathrm{H}$ methoxy) 2.381 ( $\mathrm{S}, 3 \mathrm{H}$, ethyl group), 6.023 (8H, symmulti 2 sub benzene ring).

Table 1. Elemental analysis

\begin{tabular}{|c|c|c|c|c|c|c|c|c|c|c|c|}
\hline \multirow{3}{*}{$\begin{array}{c}\text { Compd. } \\
\text { Code }\end{array}$} & \multirow{3}{*}{$\mathrm{R}$} & \multirow{3}{*}{$\begin{array}{l}\mathrm{MP} \\
{ }^{\circ} \mathrm{C}\end{array}$} & \multirow{3}{*}{ MW } & \multirow{3}{*}{$\begin{array}{l}\text { Yield } \\
\%\end{array}$} & \multirow{3}{*}{ MF } & \multicolumn{6}{|c|}{ Elemental analysis, \% } \\
\hline & & & & & & \multicolumn{2}{|c|}{$\mathrm{C}$} & \multicolumn{2}{|c|}{$\mathrm{H}$} & \multicolumn{2}{|r|}{$\mathrm{N}$} \\
\hline & & & & & & Cal & Found & Cal & Found & $\mathrm{Cal}$ & Found \\
\hline III(a) & $4 \mathrm{OCH}_{3}$ & 190 & 476 & 70 & $\mathrm{C}_{30} \mathrm{H}_{24} \mathrm{~N}_{2} \mathrm{O}_{4}$ & 75.00 & 75.00 & 9.16 & 9.10 & 5.83 & 5.82 \\
\hline III(b) & $2-\mathrm{OH}$ & 170 & 462 & 72 & $\mathrm{C}_{29} \mathrm{H}_{22} \mathrm{~N}_{2} \mathrm{O}_{4}$ & 75.00 & 74.99 & 8.62 & 8.61 & 6.03 & 6.02 \\
\hline III(c) & $2-\mathrm{Cl}$ & 200 & 480 & 70 & $\mathrm{C}_{29} \mathrm{H}_{21} \mathrm{ClN}_{2} \mathrm{O}_{3}$ & 70.02 & 70.01 & 7.64 & 7.63 & 5.63 & 5.62 \\
\hline III(d) & $4-\mathrm{Cl}$ & 200 & 480 & 71 & $\mathrm{C}_{29} \mathrm{H}_{21} \mathrm{ClN}_{2} \mathrm{O}_{3}$ & 70.02 & 70.01 & 7.64 & 7.63 & 5.63 & 5.02 \\
\hline III(e) & $4-\mathrm{OH}$ & 171 & 462 & 69 & $\mathrm{C}_{29} \mathrm{H}_{22} \mathrm{~N}_{2} \mathrm{O}_{4}$ & 75.00 & 74.99 & 8.62 & 8.61 & 6.03 & 6.02 \\
\hline III(f) & $\mathrm{H}$ & 180 & 446 & 69 & $\mathrm{C}_{29} \mathrm{H}_{22} \mathrm{~N}_{2} \mathrm{O}_{3}$ & 75.32 & 75.22 & 8.22 & 8.12 & 6.06 & 6.05 \\
\hline III $(g)$ & $\begin{array}{c}4 \mathrm{OH}, \\
2-\mathrm{OCH}_{3}\end{array}$ & 190 & 492 & 71 & $\mathrm{C}_{30} \mathrm{H}_{24} \mathrm{~N}_{2} \mathrm{O}_{5}$ & 74.84 & 74.74 & 9.56 & 9.16 & 5.82 & 5.81 \\
\hline III(h) & $4-\mathrm{CH}_{3}$ & 191 & 460 & 70 & $\mathrm{C}_{30} \mathrm{H}_{24} \mathrm{~N}_{2} \mathrm{O}_{3}$ & 77.58 & 77.48 & 9.48 & 9.46 & 6.03 & 6.02 \\
\hline III(i) & $3-\mathrm{OH}$ & 191 & 462 & 72 & $\mathrm{C}_{29} \mathrm{H}_{22} \mathrm{~N}_{2} \mathrm{O}_{4}$ & 75.00 & 74.99 & 8.62 & 8.61 & 6.03 & 6.02 \\
\hline III (j) & $\begin{array}{l}3,4,5 \\
\mathrm{CH}_{3}\end{array}$ & 170 & 488 & 71 & $\mathrm{C}_{32} \mathrm{H}_{28} \mathrm{~N}_{2} \mathrm{O}_{3}$ & 76.08 & 76.07 & 7.60 & 7.50 & 5.61 & 5.61 \\
\hline III $(k)$ & $1,2-\mathrm{NH}_{2}$ & 190 & 476 & 71 & $\mathrm{C}_{29} \mathrm{H}_{24} \mathrm{~N}_{4} \mathrm{O}_{3}$ & 74.04 & 74.03 & 9.78 & 9.68 & 11.71 & 11.81 \\
\hline III(l) & $3-\mathrm{NO}_{2}$ & 185 & 491 & 70 & $\mathrm{C}_{29} \mathrm{H}_{21} \mathrm{~N}_{3} \mathrm{O}_{5}$ & 71.31 & 71.30 & 7.78 & 7.77 & 8.60 & 8.61 \\
\hline
\end{tabular}

Antitubercular activity

All the synthesized compounds of series III(a-l) were evaluated for their antitubercular activity. Drug susceptibility and determination of MIC of the test compounds against M. tuberculosis H37Rv were performed by agar micro dilution method, where two fold 
dilutions of each test compound were added into 7H10 agars supplemented with OADC and organism. A culture of used microorganism M. tuberculosis H37Rv growing on L-J medium was harvested in $0.85 \%$ saline with $0.05 \%$ Tween-80. A suspension of compounds was prepared in DMSO. This suspension was added to (in tubes) $7 \mathrm{H} 10$ middle brook's medium (containing $1.7 \mathrm{~mL}$ medium and $0.2 \mathrm{~mL}$ OADC supplement) at different concentrations of compound keeping the volume constant, that is, $0.1 \mathrm{~mL}$ medium was allowed to cool keeping the tubes in slanting position. These tubes were then incubated at $37^{\circ} \mathrm{C}$ for 24hours followed by streaking of M. tuberculosis H37Rv $(5 \times 104$ bacilli pertube $)$. These tubes were then incubated at $37{ }^{\circ} \mathrm{C}$. Growth of bacilli was seen after 28 days of incubation. Tubes having the compounds were controlled with control tubes where medium alone was incubated with H37Rv. The concentration at which complete inhibition of colonies occurred was taken as active concentration of test compound. Isoniazid was used as standard drug. The MIC levels of compounds III(a, c, e, g, h) against these organisms were given in Table 2.

Table 2. Antitubercular activity data of the synthesized compounds (IIIa-h)

\begin{tabular}{ccc}
\hline Compound & MIC values $(\mu \mathrm{g} / \mathrm{mL})$ of M. tuberculosis $\mathrm{H}_{37} \mathrm{RV}$ & \% Inhibition \\
\hline III(a) & 62.5 & 99 \\
III(c) & 100 & 99 \\
III(e) & 75.5 & 99 \\
III(g) & 50 & 97 \\
III(h) & 25 & 99 \\
\hline
\end{tabular}

\section{Antibacterial activity}

All the compounds III(a-l) were tested for against different bacteria E.coli, B.leteus, B.fragatis, B.ovatus at two different concentration level $100 \mathrm{ppm}$ and $500 \mathrm{ppm}$ in DMF using filter paper disc diffusion method nutrient broth media. Nutrient agar and potato dextrose agars were used to culture the bacteria and fungus respectively as shown in the Table 3 and Table 4.

Table 3. Antibacterial activity data of the synthesized compounds (III a-I)

\begin{tabular}{|c|c|c|c|c|c|c|c|c|}
\hline \multirow{2}{*}{ Comp. } & \multicolumn{2}{|c|}{ B.leteus } & \multicolumn{2}{|c|}{ E.coli } & \multicolumn{2}{|c|}{ B. fragatis } & \multicolumn{2}{|c|}{ B.ovatus } \\
\hline & 100 & 500 & 100 & 500 & 100 & 500 & 100 & 500 \\
\hline III(a) & + & ++ & ++ & +++ & ++ & +++ & + & ++ \\
\hline III(b) & + & ++ & + & ++ & + & ++ & + & ++ \\
\hline III(c) & ++ & +++ & ++ & +++ & ++ & +++ & + & ++ \\
\hline $\operatorname{III}(d)$ & +++ & ++++ & ++ & +++ & ++ & +++ & ++ & +++ \\
\hline III(e) & + & ++ & + & ++ & + & ++ & + & ++ \\
\hline III(f) & - & + & - & + & + & ++ & + & ++ \\
\hline $\operatorname{III}(\mathrm{g})$ & + & ++ & + & ++ & ++ & ++ & + & ++ \\
\hline III(h) & +++ & ++++ & ++ & +++ & ++ & +++ & ++ & +++ \\
\hline III(i) & - & ++ & + & ++ & - & + & + & ++ \\
\hline $\mathbf{I I I}(\mathbf{j})$ & + & ++ & + & ++ & + & ++ & + & ++ \\
\hline III(k) & - & + & + & + & + & ++ & - & ++ \\
\hline III(I) & - & ++ & - & ++ & - & + & - & + \\
\hline Std & +++ & ++++ & +++ & ++++ & +++ & ++++ & +++ & ++++ \\
\hline
\end{tabular}

Std. Streptomycin. ++++ : strongly active range $>19 ;+++$ : moderately active range $<12-18$; ++ : weakly active range 8 -12; +,-inactive range 
Table 4. Antifungal activity data of the synthesized compounds (III a-I)

\begin{tabular}{cccccccccc}
\hline \multirow{2}{*}{ Comp. } & \multicolumn{2}{c}{ As. fusarium } & \multicolumn{2}{c}{ As. fumigates } & \multicolumn{2}{c}{ T. viridae } & \multicolumn{2}{c}{ As. Flavus } \\
\cline { 2 - 8 } & 100 & 500 & 100 & 500 & 100 & 500 & 100 & 500 \\
\hline III(a) & + & ++ & + & ++ & + & ++ & + & ++ \\
III(b) & + & ++ & + & + & ++ & ++ & + & ++ \\
III(c) & - & ++ & + & ++ & - & + & + & ++ \\
III(d) & - & + & - & + & + & ++ & + & ++ \\
III(e) & - & ++ & - & + & - & ++ & - & ++ \\
III(f) & + & ++ & - & + & + & ++ & - & + \\
III(g) & - & + & - & ++ & + & ++ & - & ++ \\
III(h) & +++ & ++++ & +++ & ++++ & ++ & +++ & +++ & ++++ \\
III(i) & - & + & + & ++ & + & ++ & + & ++ \\
III(j) & ++ & +++ & ++ & +++ & ++ & ++ & ++ & +++ \\
III(k) & + & ++ & + & ++ & + & ++ & + & +++ \\
III(l) & +++ & ++++ & +++ & ++++ & +++ & ++++ & ++ & +++ \\
Std & +++ & ++++ & +++ & ++++ & +++ & ++++ & +++ & ++++ \\
\hline
\end{tabular}

Std. Griseofulvin, ++++ : strongly active range $>19 ;+++$ : moderately active range $<12-18$; ++ : weakly active range $8-12$

\section{Acknowledgement}

The authors are thankful to Head, Department of Chemistry, Dr. H.S. Gour Central University, Sagar for providing Laboratory and IR facility we are also thankful to the Director CDRI Lucknow for providing ${ }^{1} \mathrm{H}$ NMR and Mass spectral data studies, we are also thankful to Prof. Smita Banerjee for her kind assistance for performing antimicrobial efficacy in department of Biotechnology and I also grateful to Mr. D.P. Rajani Microcare Laboratory Surat for providing anti-tubercular activity data.

\section{References}

1. Husain M I and Jamali M R, Indian J Chem., 1988, 27B, 43.

2. Colin R H and Africanpat S, Chem Abstr, 1968, 14 Nov, 6803636.

3. Mornis S, Bai G H, Suffys P, Portilo L, Gomez M and Fairchok D Rouse, J Inf Dis., 1995, 171(4), 954-960.

4. Bindra J S, Rastogi S, Patnaik G K, Anand N, Rao K, Gurudath G, Dwivedi P C and Rao C N R, Indian J Chem., 1987, 26B, 318.

5. Pasha M A, Jayashankara V P and Muhammedi K A, Indian J Chem., 2008, 47B, 1160.

6. Vartale S P, Jadhav J S, Kale M A and Kuberkar S V, Indian J Heterocycl Chem., 2006, 16, 163.

7. Kalluraya B, Nayak J and Vagdevi H M, Indian J Heterocycl Chem., 2005, 14, 257.

8. Farnanda da C, Santos Paule Abreu and Helena C Castro, Izabel C.P.P. Paixão, Claudio C. Cirne-Santos, Viveca Giongo, Juliana E. Barbosa, Bruno R. Simonetti, Valéria Garrido, Dumith Chequer Bou-Habib, David de O. Silva, Pedro N. Batalha, Jairo R. Temerozo, Thiago M. Souza, Christiane M. Nogueira, Anna C. Cunha, Carlos R. Rodrigues, Vitor F. Ferreira and Maria C.B.V. de Souza, Bioorg Med Chem., , 2009, 17(15), 5476-5481. ,

9. Coatney G R, Am J Trop Med Hyg., 1963, 12, 121-128. 
10. A Bunger in Relation of Chemical structure and biological activity, Medicinal Chemistry, $3^{\text {rd }}$ Ed., Wiley Interscience, 1970, 72.

11. French F A and Blanz E J, Cancer Res., 1965, 25, 1454-1458.

12. Matloo B N, Trans Faraday Soc., 1956, 52, 1184.

13. Ambica Shrivastava, Mrityunjay K Singh and Singh R M, Indian J Chem., 2006, 45B, 292-296.

14. Abha Bisnai, Krishna Shrivastava and Joshi M N, Indian J Chem., 2006, 45B, 1961-1964.

15. Yusuf M. Al-Hiari, Rana Abu-Dahab and Mustafa M. El-Abadelah, Molecules, 2008, 13(11), 2880-2893.

16. Soňa Jantová, Silvia Letašiová, Katarína Konariková, Viktor Milata and Vlasta Brezová, Acta Chimica Slovaca, 2010, 3(1), 51-72.

17. Nakamura S, Minami A, Nakata K, Kurobe N, Kouno K, Sakaguchi Y, Kashimoto S, Yoshida H, Kojima T and Ohue T, Antimicrob Agents Chemotherapy, 1989, 33(8), 1167-1173.

18. Sundra Amrini, Sandra Fillippi, Astria Parenti, Fabrizio Ledda and Marina Ziche, British J Pharmacol., 1997, 122, 1739.

19. Bauer I, Max N, Fatzer S and Lingeni F, Eur J Biochem., 1996, 240, 576.

20. (a) Anargyros P, David S J, Irene A and Lim I L, J Clin Microbiol., 1990, 28, 1288-1291.

21 Rattan A, Antimicrobials in Laboratory Medicine, $5^{\text {th }}$ Ed., Churchill Livingstone B Y, New Delhi, 2005, pp. 85-90. 